\title{
Rearrangement of Nucleosomal Components by Modification of Histone Amino Groups. Structural Role of Lysine Residues ${ }^{\dagger}$
}

\author{
Juan Jordano, Francisco Montero, and Enrique Palaciån*
}

\begin{abstract}
Modification of nucleosomal particles from chicken erythrocytes with the reagents for protein amino groups acetic and dimethylmaleic anhydrides causes a rearrangement of nucleosomal components. Treatment with both reagents is accompanied by liberation of free DNA and formation of residual particles with anomalous histone composition. The residual particles obtained with acetic anhydride contain an excess of histones corresponding to the free DNA produced. In contrast, dimethylmaleic anhydride causes release of histones $\mathrm{H} 1, \mathrm{H} 5, \mathrm{H} 2 \mathrm{~A}$ and $\mathrm{H} 2 \mathrm{~B}$ and formation of residual particles deficient in these histones but containing an excess of $\mathrm{H} 3$ and $\mathrm{H} 4$ corresponding to the liberated DNA. Regen-
\end{abstract}

\section{D} ouble-stranded DNA is wrapped around a histone octamer to form the nucleosomal core which is the basic structure present in chromatin (McGhee \& Felsenfeld, 1980; Sperling $\&$ Wachtel, 1981). The forces that distort the DNA double helix to produce a condensed superhelical structure appear to be essentially electrostatic. The negatively charged phosphate groups of DNA would interact with the positively charged amino acid residues, lysine and arginine, of the core histones to induce the nucleosomal DNA structure. Arginine-rich histones, $\mathrm{H} 3$ and $\mathrm{H} 3$, are electrostatically more strongly bound to DNA than the lysine-rich pair, $\mathrm{H} 2 \mathrm{~A}$ and $\mathrm{H} 2 \mathrm{~B}$, the two first histones being essential for the organization of DNA structure in nucleosomes (Camerini-Otero et al., 1976; Sollner-Webb et al., 1976; Camerini-Otero \& Felsenfeld, 1977; Daban \& Cantor, 1982).

Recently, an essential role in the folding of DNA has been assigned to a small number of arginine residues (Ichimura et al., 1982). Interaction of lysine residues with DNA, although not as strong as that of arginine, should contribute to the stabilization of nucleosomal cores, as indicated by the low reactivity of nucleosomal lysines toward the reagent $\mathrm{AA}^{1}$ (Malchy \& Kaplan, 1974). The small effect on chromatin structure of extensive modification of lysine residues with ethyl acetimidate, which produces no change in electric charge, as compared to the large effects obtained with AA, which eliminates the positive charge of amino groups, stresses and importance of the electrostatic interactions between lysine residues and DNA (Tack \& Simpson, 1977). An experimental approach to investigate the role of lysine residues in nucleosomal structure is the chemical modification of these residues followed by determination of the structural changes produced. We have used DMMA in addition to AA. The first reagent

\footnotetext{
${ }^{\dagger}$ From the Centro de Biologia Molecular, Consejo Superior de Investigaciones Cientificas y Universidad Autónoma de Madrid, Canto Blanco, Madrid-34, Spain (J.J. and E.P.), and the Departamento de Bioquimica, Facultad de Ciencias Quimicas, Universidad Complutense, Madrid-3, Spain (F.M.). Received November 2, 1983. This work was supported in part by the Fondo de Investigaciones Sanitarias and the Comisiōn Asesora de Investigaciôn Científica y Técnica (Spain).
}

eration of the modified amino groups of nucleosomal preparations treated with dimethylmaleic anhydride is accompanied by reconstitution of nucleosomal particles with the sedimentation coefficient and composition of core histones of the original nucleosomes. This reconstitution does not occur when the released fraction containing histones $\mathrm{H} 2 \mathrm{~A}$ and $\mathrm{H} 2 \mathrm{~B}$ and free DNA is separated from the residual particles. The studied disassembly of nucleosomal particles obtained by specifically blocking lysine-DNA interactions with these reagents appears to indicate that lysine residues are essential for the binding of DNA to histones with formation of nucleosomal particles.

causes an electrostatic change larger than the second by introducing a negatively charged residue instead of the uncharged acetyl group of AA (Means \& Feeney, 1971). Modification of oligomeric proteins or protein-containing particles with DMMA is frequently accompanied by dissociation of protein components (Means \& Feeney, 1971; Pintor-Toro et al., 1979, 1982; Vioque et al., 1982). Since the DMMA-modified amino groups can be easily regenerated at moderately acid pH (Dixon \& Perham, 1968), the dissociated fraction as well as the residual particles can be isolated in unmodified form, and the original particles can be potentially reconstituted (Pintor-Toro et al., 1981; Vioque et al., 1982). These properties make DMMA a useful reagent to study the contribution of lysine residues to the building of protein-containing particles such as nucleosomes.

In this paper, we study the effects on nucleosome integrity and structure produced by blocking lysine-DNA interactions with AA and the reversible reagent DMMA. The observed disassembly and rearrangement of nucleosomal particles induced by the modification are indicative of the essential contribution of lysine-DNA interactions to DNA folding in the nucleosomal core.

\section{Materials and Methods}

Preparation of Nucleosomal Particles. Nuclei were obtained from chicken erythrocytes after lysis of the cells in a buffer solution containing $0.5 \%$ of the nonionic detergent Nonidet P-40 (Weintraub et al., 1975). Mononucleosomes were prepared by digestion of the isolated nuclei with micrococcal nuclease (Worthington) and fractionation of the digestion products by centrifugation on a 5-20\% linear sucrose gradient, essentially as described by Lacy \& Axel (1975). The concentration of nucleosomal particles was determined spectrophotometrically, taking $A_{260 \mathrm{~nm}}=20.0$ for a solution con-

1 Abbreviations: AA, acetic anhydride; DMMA, dimethylmaleic anhydride; PMSF, phenylmethanesulfonyl fluoride; EDTA, ethylenediaminetetraacetic acid; Tris- $\mathrm{HCl}$, tris(hydroxymethyl)aminomethane hydrochloride. 
taining $1.0 \mathrm{mg}$ of DNA $/ \mathrm{mL}$. Nucleosomal DNA had an average length of $160 \pm 10$ base pairs as compared with the digestion products of plasmid $\mathrm{pBR} 322$ treated with nuclease HaeIII.

Modification of Nucleosomes with Carboxylic Acid Anhydrides. Nucleosomes $(0.2-0.4 \mathrm{mg}$ of DNA $/ \mathrm{mL})$, in $10 \mathrm{mM}$ 4-(2-hydroxyethyl)-1-piperazineethanesulfonic acid $\left(\mathrm{K}^{+}\right)(\mathrm{pH}$ 8.2), $5 \mathrm{mM}$ EDTA, and 0.1 mM PMSF, were treated at room temperature with the desired amount of AA or DMMA in dioxane. During reaction the $\mathrm{pH}$ was maintained at 8.2 by addition of $0.5 \mathrm{~N} \mathrm{KOH}$. The hydrolyzed reagent was eliminated by overnight dialysis at $2-4^{\circ} \mathrm{C}$ against $10 \mathrm{mM}$ Tris$\mathrm{HCl}$ ( $\mathrm{pH}$ 8.2), $5 \mathrm{mM}$ EDTA, and $0.1 \mathrm{mM}$ PMSF. An estimation of the number of lysine residues modified by DMMA was made by determining the lysine residues that react with trinitrobenzenesulfonic acid in the DMMA-treated preparation and the untreated control, as described by Ichimura et al. (1982).

Regeneration of the Modified Amino Groups and Reconstitution of Nucleosomal Particles. The modified amino groups were regenerated by dialysis of the DMMA-treated preparations at $2-4{ }^{\circ} \mathrm{C}$ for $6 \mathrm{~h}$ against $10 \mathrm{mM}$ maleate $(\mathrm{pH}$ 6.0), $1 \mathrm{M} \mathrm{NaCl}, 5 \mathrm{mM}$ EDTA, and $0.1 \mathrm{mM}$ PMSF. The regenerated preparations were dialyzed against $10 \mathrm{mM}$ Tris- $\mathrm{HCl}$ (pH 8.2), $5 \mathrm{mM}$ EDTA, and 0.1 mM PMSF. When the regeneration procedure was applied to preparations containing all the nucleosomal components, reconstituted nucleosomes were formed which could be isolated by centrifugation in 5-20\% sucrose gradients.

Sedimentation Analysis. Sedimentation was studied by centrifugation in linear 5-20\% sucrose gradients containing $10 \mathrm{mM}$ Tris- $\mathrm{HCl}(\mathrm{pH} \mathrm{8.2),} 5 \mathrm{mM}$ EDTA, and $0.1 \mathrm{mM}$ PMSF. The nucleosomal preparations $(0.2-0.4 \mathrm{mg}$ of DNA) were centrifuged in a Beckman SW40 rotor at $30000 \mathrm{rpm}$ and $6^{\circ} \mathrm{C}$ for $26 \mathrm{~h}$. The distribution of particles along the gradients was determined with an ISCO density fractionator. Sedimentation coefficients were obtained in a Beckman analytical ultracentrifuge (Model E), at $56000 \mathrm{rpm}$ and $8^{\circ} \mathrm{C}$, using the An-F rotor and double sector cells. Sedimentation was followed with a photoelectric scanner at $260 \mathrm{~nm}$. The sample, in $10 \mathrm{mM}$ Tris- $\mathrm{HCl}(\mathrm{pH} 8.2), 5 \mathrm{mM}$ EDTA, and $0.1 \mathrm{mM}$ PMSF, contained $40 \mu \mathrm{g}$ of DNA $/ \mathrm{mL}$.

Electrophoretic Analysis of Histones and DNA. Samples were dialyzed against $0.5 \%$ acetic acid and lyophilized prior to electrophoresis of histones. Electrophoresis was conducted on polyacrylamide gels prepared from $15 \%$ acrylamide and containing $0.1 \%$ sodium dodecyl sulfate according to Laemmli (1970). Histones in the gels were stained with a solution containing $0.05 \%$ Coomassie Blue, $7.5 \%(\mathrm{v} / \mathrm{v})$ acetic acid, and $10 \%(\mathrm{v} / \mathrm{v})$ ethanol. Densitometric analysis of the electrophoretic patterns was performed with an Optronic digital densitometer conected to a PDP11/45 computer. DNA was isolated as described by Marmur (1961) and subjected to electrophoresis on mixed gels containing $0.5 \%$ agarose and $3 \%$ polyacrylamide according to Peacock \& Dingman (1967).

\section{Results}

When nucleosomal particles are treated with DMMA, a reagent for protein amino groups, a change in the sedimentation properties of the particles is produced. Figure 1 shows the sedimentation patterns of preparations treated with different amounts of reagent. Two components with sedimentation coefficients smaller than that of the original particles are observed. Since the sedimentation patterns were obtained by measuring the absorbance at $254 \mathrm{~nm}$, a wavelength at which the relative absorbance of histones is negligible, the size of the

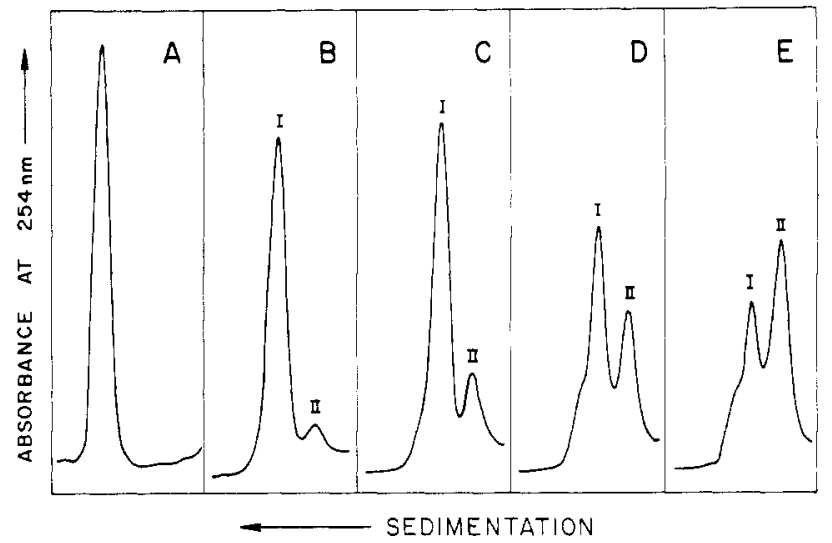

FIGURE 1: Sedimentation patterns of nucleosomal preparations treated with different amounts of DMMA. Nucleosomal particles were treated with the following amounts of DMMA (mg/mL): 0.29 (B), 0.58 (C), 1.16 (D), and $1.74(\mathrm{E})$. Prior to sucrose gradient centrifugation, the hydrolyzed reagent was eliminated by dialysis as indicated under Materials and Methods. (A) is a control subjected to the same treatments as the modified preparations but in the absence of DMMA.

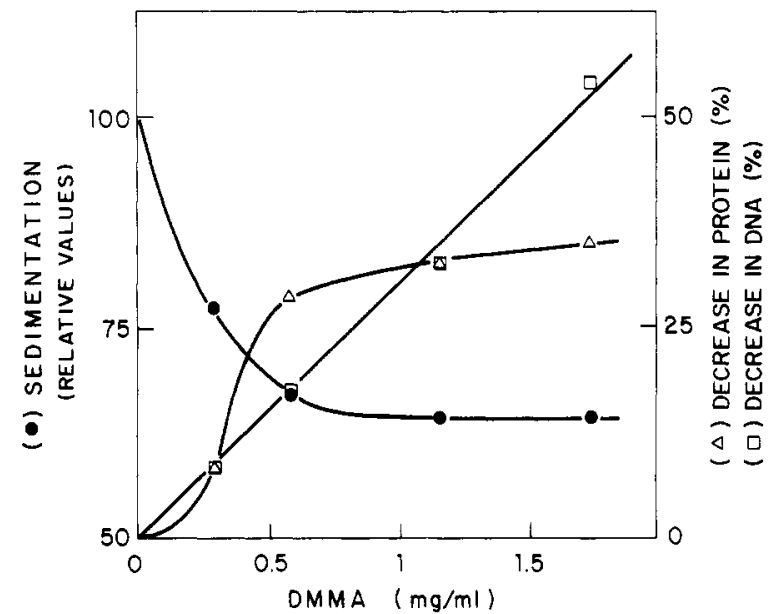

FIGURE 2: Sedimentation of component I and decrease in its protein and DNA content after different DMMA treatments. The preparations are the same used for the experiment shown in Figure 1. Sedimentation is expressed as the distance from the peak of component I to the meniscus relative to that corresponding to the untreated nucleosomal particles, to which a value of 100 is assigned. The decrease in the amount of DNA in component I was estimated from the areas of components I and II in the sedimentation patterns shown in Figure 1. The protein content was evaluated by densitometry of the electrophoretic patterns obtained from component I and the released fraction (Figure 3).

peaks corresponds to the amount of DNA present. With increasing DMMA treatments there is a loss of material from the fast component (component I) and a corresponding increase in the slow one (component II). The rate of sedimentation of component I decreases with the increase in the amount of reagent used until it approaches a plateau (Figure 2 ), while that of component II remains practically unchanged at all levels of DMMA.

To further identify components I and II obtained with DMMA, electrophoresis of the corresponding DNA fractions and of the histones in peak $I$ and in the released fraction (from the end of peak I to the meniscus) was carried out. It was found that the DNA in both components has the same length as the DNA in the original preparation. Figure 3 shows the electrophoresis of the histones present in component $I$ and in the released fraction of the preparations studied in the experiment of Figure 1. Modification is accompanied by release of histones $\mathrm{H} 1, \mathrm{H} 5, \mathrm{H} 2 \mathrm{~A}$, and $\mathrm{H} 2 \mathrm{~B}$, while $\mathrm{H} 3$ and $\mathrm{H} 4$ remain bound in the residual particles even at the highest level of 


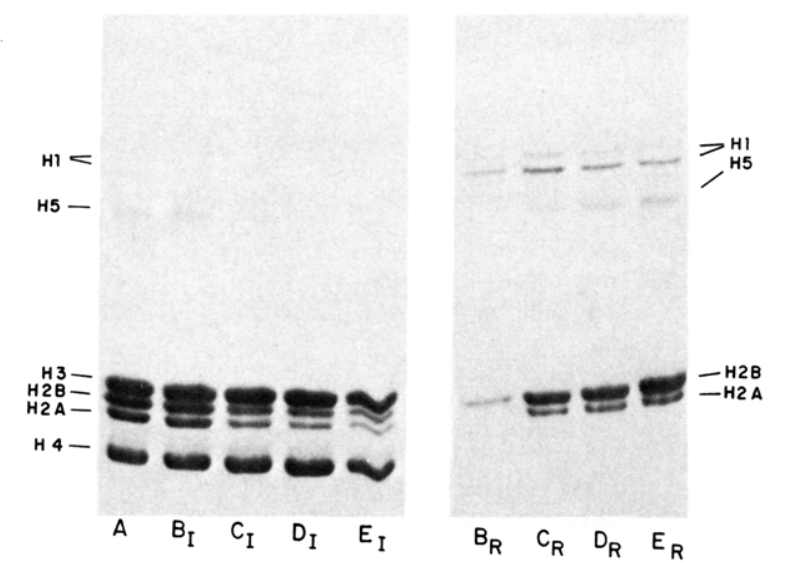

FIGURE 3: Electrophoresis of the histones released and of those present in component I at different DMMA treatments. The preparations are the same used for the experiments shown in Figures 1 and 2. Samples $B_{1}-E_{I}$ correspond to component I of preparations B-E described in Figure 1, while samples $B_{R}-E_{R}$ are the released fractions of preparations B-E. Sample A is the untreated control. All the DMMA-treated samples correspond to the same amount of the original preparation, while sample $\mathbf{A}$ is present in a smaller amount.

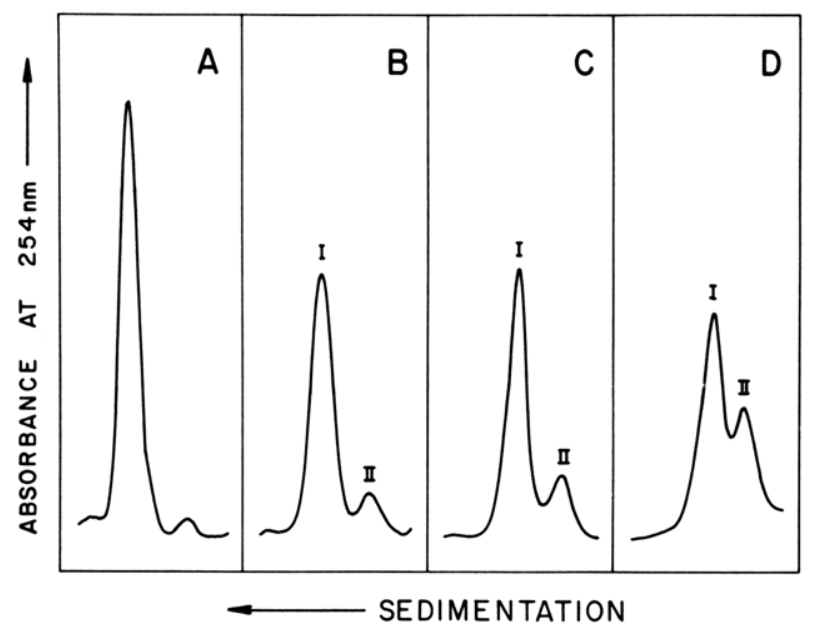

FIGURE 4: Sedimentation patterns of nucleosomal preparations treated with different amounts of AA. Nucleosomal particles were treated with the following amounts of AA $(\mathrm{mg} / \mathrm{mL}): 0.12(B), 0.25(\mathrm{C})$, and 0.82 (D). Prior to sucrose gradient centrifugation, the hydrolyzed reagent was eliminated by dialysis. (A) is a control subjected to the same treatments as the modified preparations but in the absence of AA.

DMMA used. The released proteins are distributed in the gradient from the end of peak I to the meniscus, not being concentrated in peak II which has the same concentration of protein as the fraction near the meniscus, indicating that the liberated histones do not appear to be associated to the DNA of peak II.

The loss of DNA from component I is linear with respect to the amount of DMMA used, in contrast with the pattern of protein release (Figure 2). Part of the protein present in the particles that dissociate to produce free DNA binds to the remaining residual particles, as shown from analysis of component I (Figure 3). Liberation of histones from fraction I takes place in two steps: there is a sudden release followed by a slow increase in the total amount liberated. This pattern of release is similar to that obtained for histones H2A and H2B.

Treatment of nucleosomal particles with AA is also accompanied by a change in sedimentation pattern (Figure 4) similar to that produced by DMMA, with transfer of DNA from component I to component II in response to increasing
Table I: Sedimentation Coefficients of Different Nucleosomal Particles $^{a}$

\begin{tabular}{lcc}
\hline \multicolumn{1}{c}{ particles } & $\begin{array}{c}\text { gradient } \\
\text { sedimentation } \\
\text { (rel values) }\end{array}$ & $\begin{array}{c}\text { sedimentation } \\
\text { coefficient } \\
s_{20}(\mathrm{~S})\end{array}$ \\
\hline original nucleosomes & 100.0 & $10.8^{b}$ \\
reconstituted particles & 97.0 & 10.5 \\
component I (DMMA) & 84.0 & 8.9 \\
& 70.5 & $7.4^{b}$ \\
component II (DMMA) & 62.0 & 6.0 \\
component II (AA) & 36.0 & $2.7^{b}$ \\
\hline
\end{tabular}

${ }^{a}$ The relative values of sedimentation in sucrose gradients were obtained as indicated in the legend of Figure 2. ${ }^{b}$ These sedimentation coefficients were determined in the analytical ultracentrifuge. They were used to calibrate the sucrose gradient runs and to obtain approximate sedimentation coefficients for the other particles shown.

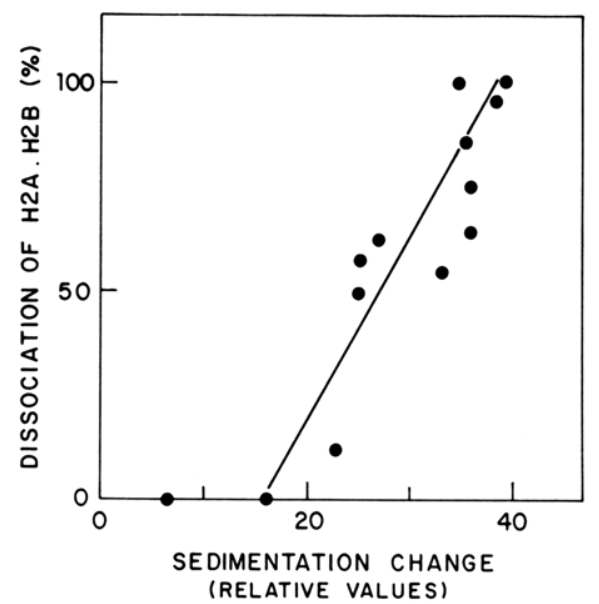

FIGURE 5: Dissociation of histones $\mathrm{H} 2 \mathrm{~A}$ plus $\mathrm{H} 2 \mathrm{~B}$ and change in sedimentation of component $\mathrm{I}$. The extent of dissociation of $\mathrm{H} 2 \mathrm{~A}$ plus H2B was evaluated by densitometry of electrophoresis patterns like those in Figure 3. Sedimentation change is expressed as the difference between the sedimentation of the original nucleosomal particles and that of component I, obtained as indicated in the legend to Figure 2.

amounts of reagent, but without release of protein from fraction I. Analysis by electrophoresis of components I and II showed that all the histone present in the preparation is associated to peak I, while no protein is detected in peak II. The lack of histone release from fraction I with AA is explained by the lower distortion of the electrostatic interactions that this reagent should produce as compared with that of DMMA. Both components II obtained with either AA or DMMA have similar sedimentation coefficients (Table I).

The series of preparations corresponding to the experiments shown in Figures 1-3 is representative of the process studied. However, there are variations among different experiments that might depend on the particular nucleosomal preparation modified. Sometimes, the amount of reagent needed to obtain a certain change in sedimentation and a fixed degree of $\mathrm{H} 2 \mathrm{~A} \cdot \mathrm{H} 2 \mathrm{~B}$ dissociation is slightly different. In addition, the degree of liberation of histones $\mathrm{H} 2 \mathrm{~A}$ and $\mathrm{H} 2 \mathrm{~B}$ can reach $100 \%$. The extent of dissociation of these proteins is more directly related to the change in sedimentation obtained (Figure 5) than to the amount of DMMA added. In contrast, the percentage of DNA present in component $I$ is linearly dependent on the amount of reagent used, as shown in Figure 6 . These results are in agreement with the lack of correlation between DNA release and the liberation of histones from the fraction of residual particles.

An estimation of the number of protein amino groups that react with DMMA was made by determination of the groups 


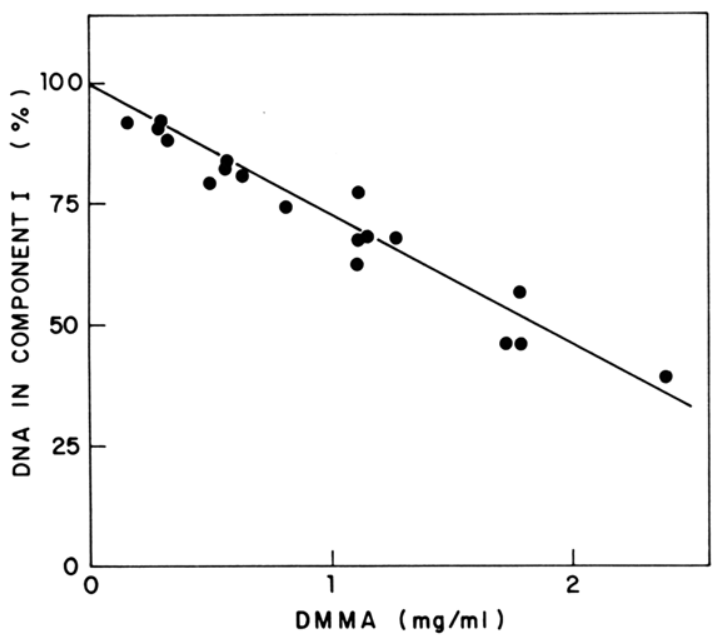

FIGURE 6: Percentage of DNA present in component I for different DMMA treatments. DNA present in component I was estimated from the areas of components I and II in sedimentation patterns like those shown in Figure 1.

in the modified preparation that react with trinitrobenzenesulfonic acid. Of the 78 amino groups that react with this compound in the nucleosome core $(67 \%$ of all the lysine residues in the histone octamer) (Ichimura et al., 1982), approximately 40 are blocked by DMMA for a treatment producing a relative decrease in sedimentation of $37 \%$ as compared with that of the native nucleosomes.

The change in sedimentation of the residual particles takes place in two steps. The first step in DMMA treatment causes a weakening of the interactions between histones and DNA, which allows a slight uncoiling of DNA and therefore produces a particle with a decreased sedimentation coefficient (Figure 5 and Table I). The second step is accompanied by release of histones H2A and H2B and results in a much larger change in sedimentation (Figure 5 and Table I).

Since the protein amino groups modified by DMMA can be regenerated at moderately acid $\mathrm{pH}$, nucleosomal particles similar to the original might be reconstituted from preparations modified by DMMA. Figure 7 shows the sedimentation pattern of a DMMA-treated preparation after regeneration of the modified amino groups by incubation at $\mathrm{pH} 6.0$. A significant fraction of the treated material migrates in a symmetrical peak with a sedimentation coefficient almost identical with that of the original nucleosomes (Table I). In contrast, regeneration of component $I$ in the absence of the released fraction produces particles sedimenting more slowly than the native nucleosomes. These results indicate that to obtain a correct reconstitution all the components of nucleosomal particles must be present. In particular, histones H2A and H2B should be added, since component $I$ is deficient in these core histones.

The reconstituted particles obtained from a DMMA-treated preparation in which $100 \%$ of histones $\mathrm{H} 2 \mathrm{~A}$ and $\mathrm{H} 2 \mathrm{~B}$ has been dissociated have the same core histone composition as the native particles (Figure 8). Reconstituted particles show sometimes a slight excess of protein over DNA as compared with native nucleosomes, this excess being not higher than $20 \%$. In contrast, histones $\mathrm{H} 1$ and $\mathrm{H} 5$ are less efficiently incorporated into the reconstituted particles, which are therefore deficient in these proteins with respect to the untreated control.

\section{Discussion}

Treatment of nucleosomal particles with AA or DMMA, reagents for protein amino groups, is accompanied by a rearrangement of nucleosomal components. The modified

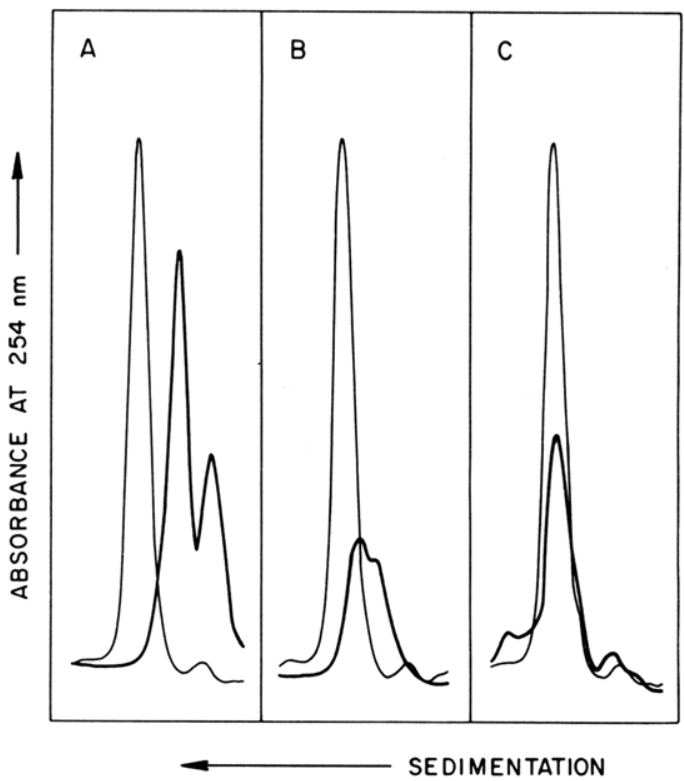

FIGURE 7: Recovery of the original sedimentation pattern upon regeneration of the modified amino groups. The nucleosomal particles were treated with $1.12 \mathrm{mg}$ of DMMA/mL. (A) Modified preparation; (B) component I, isolated from the modified preparation, after regeneration of the modified amino groups; (C) DMMA-treated preparation after regeneration of amino groups. The superimposed thin tracings correspond to the original nucleosomal particles.

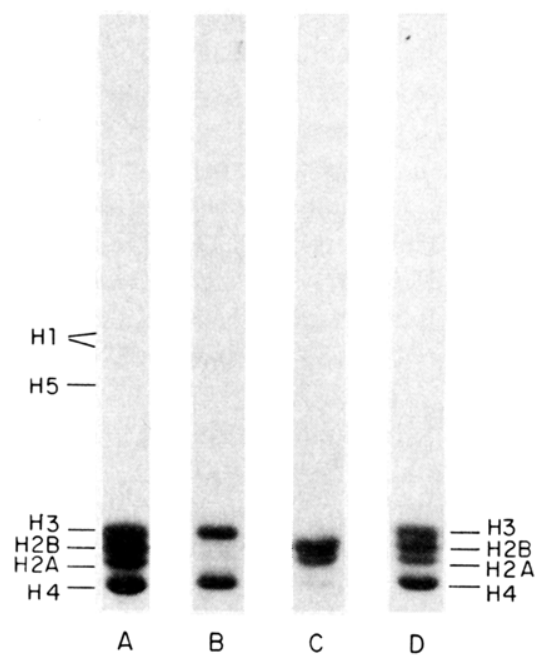

FIGURE 8: Histone composition of the particles obtained after regeneration of the modified amino groups. The nucleosomal particles were treated with $1.12 \mathrm{mg}$ of DMMA $/ \mathrm{mL}$. (A) Untreated nucleosomal particles; (B) component I; (C) released fraction; (D) reconstituted particles isolated from the regenerated preparation by density gradient centrifugation.

particles obtained with AA contain an excess of histone octamer $(\mathrm{H} 2 \mathrm{~A} \cdot \mathrm{H} 2 \mathrm{~B} \cdot \mathrm{H} 3 \cdot \mathrm{H} 4)_{2}$, while those prepared with DMMA are deficient in histones $\mathrm{H} 2 \mathrm{~A}$ and $\mathrm{H} 2 \mathrm{~B}$, having an excess of $\mathrm{H} 3$ and $\mathrm{H} 4$. With both reagents free DNA is released. The loss of DNA is not dependent on the release of $\mathrm{H} 2 \mathrm{~A}$ and H2B from the fraction of residual particles, since treatments with AA that do not produce liberation of these proteins are nevertheless accompanied by a release of DNA similar to that obtained with DMMA.

In contrast with high ionic strength, which decreases the electrostatic interactions between basic histone residues (lysine and arginine) and DNA, modification with DMMA or AA affects the interaction of lysine only, which potentially allows an evaluation of the role played by this residue in the binding of DNA to the histone octamer. Nevertheless, the residues 
introduced by modification may have other effects on nucleosomal structure in addition to the disruption of lysineDNA interactions. Among these effects we can include steric distortion caused by the reagent moieties introduced and, in the case of modification with DMMA, electric repulsion between the negatively charged moieties and other negative portions of the particle, especially the phosphate groups of DNA. The steric distortion produced by AA appears to be minimal, since extensive modification of chromatin with ethyl acetimidate, which introduces a residue of a size similar to that of AA but contains a positive charge, is not accompanied by any significant change in structure (Tack \& Simpson, 1977).

The release of free DNA as a result of modification with AA or DMMA seems to indicate that the interaction of the lysine residues of core histones with DNA is essential for the binding of DNA to histones to make up the nucleosomal core particle. However, these same residues are not necessary for the binding of histones $\mathrm{H} 3$ and $\mathrm{H} 4$ or a whole histone octamer, as extra components, on nucleosomal cores. It is reasonable to conclude that, in addition to the interactions between arginine residues and DNA, those of lysines with DNA seem to be essential for the folding of DNA into a nucleosomal core structure. Since liberation of free DNA from DMMA-treated particles lacking H2A and H2B takes place on further modification (results not shown), arginine-rich histones $\mathrm{H} 3$ and $\mathrm{H} 4$ appear to contain lysine residues, the modification of which is accompanied by dissociation of free DNA.

Regeneration of the lysine residues of DMMA-modified preparations is accompanied by reconstitution of nucleosomal particles which have the sedimentation coefficient and core histone composition of the original nucleosomal particles. To obtain reconstitution, the presence of the released fraction, in addition to the residual particles, is required. The released fraction supplies the dissociated histones $\mathrm{H} 2 \mathrm{~A}$ and $\mathrm{H} 2 \mathrm{~B}$ and free DNA. H2A and $\mathrm{H} 2 \mathrm{~B}$ are needed to complement the residual particles which are deficient in these histones, while free DNA might be required to bind the excess $\mathrm{H} 3$ and $\mathrm{H} 4$ present in the residual particles.

In summary, modification of the lysine residues of nucleosomes causes liberation of free DNA and the formation of residual particles with anomalous histone composition. The availability of $\mathrm{H} 2 \mathrm{~A} \cdot \mathrm{H} 2 \mathrm{~B}$-deficient particles and the convenient procedures to regenerate their modified amino groups and to reconstitute nucleosomal particles should facilitate the study of the contribution of histones $\mathrm{H} 2 \mathrm{~A}$ and $\mathrm{H} 2 \mathrm{~B}$ to the overall nucleosomal core structure. In the following paper (Jordano et al., 1984), we try to answer this question by studying the structural properties of the residual as well as the reconstituted particles, making use of DNase I digestion analysis, circular dichroism, and thermal denaturation.

\section{Acknowledgments}

We thank Dr. Juan Modolell for critical reading of the manuscript and María José Chuliá for technical assistance.

Registry No. AA, 108-24-7; DMMA, 766-39-2; lysine, 56-87-1.

References

Camerini-Otero, R. D., \& Felsenfeld, G. (1977) Nucleic Acids Res. 4, 1159-1181.

Camerini-Otero, R. D., Sollner-Webb, B., \& Felsenfeld, G. (1976) Cell (Cambridge, Mass.) 8, 333-347.

Daban, J. R., \& Cantor, C. R. (1982) J. Mol. Biol. 156, 771-789.

Dixon, H. B. F., \& Perham, R. N. (1968) Biochem. J. 109, 312-314.

Ichimura, S., Mita, K., \& Zama, M. (1982) Biochemistry 21, 5329-5334.

Jordano, J., Montero, F., \& Palaciăn, E. (1984) Biochemistry (following paper in this issue).

Lacy, E., \& Axel, R. (1975) Proc. Natl. Acad. Sci. U.S.A., 72, 3978-3982.

Laemmli, U. K. (1970) Nature (London) 227, 680-685.

Malchy, B., \& Kaplan, H. (1974) J. Mol. Biol. 82, 537-545.

Marmur, J. (1961) J. Mol. Biol. 3, 208-218.

McGhee, J. D., \& Felsenfeld, G. (1980) Annu. Rev. Biochem. 49, 1115-1156.

Means, G. E., \& Feeney, R. E. (1971) Chemical Modification of Proteins, Holden-Day, San Francisco.

Peacock, A. C., \& Dingman, C. W. (1967) Biochemistry 6, 1818-1827.

Pintor-Toro, J. A., Vázquez, D., \& Palaciān, E. (1979) Biochemistry 18, 3219-3223.

Pintor-Toro, J. A., Hernāndez, F., Lōpez-Rivas, A., \& Palaciăn, E. (1981) Arch. Biochem. Biophys. 210, 786-789.

Pintor-Toro, J. A., Hernāndez, F., Löpez-Rivas, A., \& Palaciän, E. (1982) Mol. Cell. Biochem. 43, 43-47.

Sollner-Webb, B., Camerini-Otero, R. D., \& Felsenfeld, G. (1976) Cell (Cambridge, Mass.) 9, 179-193.

Sperling, R., \& Wachtel, E. J. (1981) Adv. Protein Chem. 34, $1-60$.

Tack, L. O., \& Simpson, R. T. (1977) Biochemistry 16, 3746-3753.

Vioque, A., Pintor-Toro, J. A., \& Palaciản, E. (1982) J. Biol. Chem. 257, 6477-6480.

Weintraub, H., Palter, K., \& Van Lente, F. (1975) Cell (Cambridge, Mass.) 6, 85-100. 